\title{
Kinetic Theory of Hydrodynamic Flows, III. The Torque on a Rotating Sphere or Cylinder ${ }^{1}$
}

\author{
Henk van Beijeren ${ }^{2}$ and J. R. Dorfman ${ }^{3}$
}

Received April 2, 1982

\begin{abstract}
The extended Boltzmann equation introduced in previous papers is used to compute the torque exerted on a macroscopic sphere or cylinder placed in a dilute gas, when the mean free path of the gas molecules is small compared to the characteristic dimension of the sphere or cylinder. The usual hydrodynamic results are recovered in this kinetic theory calculation.
\end{abstract}

KEY WORDS: Boltzmann equation; boundary conditions; normal solutions; rotating sphere or cylinder; torque.

\section{INTRODUCTION}

In two earlier papers, ${ }^{(1,2)}$ referred to here as I and II, respectively, we showed how to calculate the drag force on a macroscopic sphere or cylinder moving through a dilute gas by using an extended Boltzmann equation. This equation differs from the usual Boltzmann equation in that it treats the effect of collisions between gas molecules and the macroscopic object as a term in the Boltzmann equation itself rather than by means of a boundary condition to be satisfied by the distribution function at the surface of the object. This equation, although equivalent to the Boltzmann equation plus boundary conditions, ${ }^{(3)}$ enabled us to describe the gas flow in both the rarefied and hydrodynamic regions and to compute the drag force directly in terms of the collisions between the gas molecules and the object.

\footnotetext{
1 Work supported in part by the National Science Foundation Grant No. CHE 77-16308.

${ }^{2}$ Institute for Theoretical Physics, R. W. T. H., Aachen, West Germany.

${ }^{3}$ Institute for Physical Science and Technology and Department of Physics and Astronomy, University of Maryland, College Park, Maryland 20742.
} 
Two equivalent methods were used to compute the drag force for the hydrodynamic regime, where the size of the object is much larger than the mean free path of the gas molecules. The first method, used in I, could be viewed as an extension of the Chapman-Enskog normal solution of the Boltzmann equation that also takes into account the kinetic boundary layer that forms around the object, whereas the second method, used in II, was an iterated solution of the extended Boltzmann equation that explicitly displayed the dynamical events responsible for the drag force.

In this paper we use these methods to compute the rotational drag, or torque, on a large sphere or cylinder that is rotating about a body axis of symmetry in a dilute gas. We consider only the torque on the object in the hydrodynamic regime since that is a more stringent test of our method of solution of the extended Boltzmann equation than the torque in the rarefied regime, although the methods of II could easily be extended to treat that case also. We will compare our results with expressions for the torque on a sphere or cylinder that have been derived from the solutions of the linearized Navier-Stokes equations of continuum hydrodynamics. ${ }^{(4,5)} \mathrm{We}$ will see that our results agree with those of hydrodynamics if the transport coefficients in the hydrodynamic equations are replaced by their lowdensity values.

In addition to illustrating the microscopic processes responsible for the properties of hydrodynamic flows, the results derived here can be related to recent work on nonequilibrium properties of dense fluids. There has been a considerable amount of work during the past several years on the application of hydrodynamic methods to compute the time correlation functions that determine transport coefficients for a fluid or the friction coefficients for a Brownian particle moving in the fluid. Much of this work has been summarized in recent reviews. ${ }^{(6-8)}$ Of particular interest to us here is the work by Hauge and Martin Löf, ${ }^{(9)}$ by Hynes, Kapral, and Weinberg, ${ }^{(10)}$ and by Lebenhaft and Kapral ${ }^{(1)}$ on the rotational friction coefficient for a Brownian particle in a fluid. All of these authors consider that the fluid surrounding the Brownian particle satisfies the linearized Navier-Stokes equations, and that the fluid is coupled to the Brownian particle either through boundary conditions satisfied by the fluid variables at the surface of the particle ${ }^{(9,10)}$ or through source terms appearing in the hydrodynamic equations that treat the Brownian particle as a source of momentum, angular momentum, and energy for the fluid. ${ }^{(11)}$ Translational and rotational frictional coefficients as well as velocity and angular velocity correlation functions for the Brownian particle are then calculated by solving the hydrodynamic equations and performing averages over fluctuations as in Ref. 9 or over appropriate ensembles, as in Refs. 10 and 11. In any case 
these authors derive expressions for the rotational friction coefficient which agree with the results obtained here for the rotational friction on a large macroscopic sphere. The work of Lebenhaft and Kapral, ${ }^{(11)}$ in particular, is closest in spirit to that described here, and is designed to shed some light on the molecular processes that underlie the phenomena of Brownian motion.

The plan of this paper is as follows: In Section 2 we consider the extended Boltzmann equation for a dilute gas in which there is a large macroscopic sphere. We suppose that the gas-object collisions can be described by a Maxwell collision operator which is a linear combination of an operator that describes specular collisions and one that describes diffusive collisions. The combination is specified by an accommodation coefficient $\alpha$. We then suppose the macroscopic sphere undergoes a rotation about a fixed diameter and compute the torque on the sphere as a function of the coefficient $\alpha$ by applying the generalized Chapman-Enskog solution developed in Paper I to the case of interest here. In Section 3 we consider the object to be a large cylinger rotating about its long axis of symmetry and use similar methods to compute the torque that the gas exerts on it. We conclude with a brief discussion of some further applications of this work.

\section{THE ROTATING SPHERE}

As in the previous papers our starting point is the extended Boltzmann equation, which is given by ${ }^{(1)}$

$$
\frac{\partial F}{\partial t}(\mathbf{r}, \mathbf{v}, t)+\mathbf{v} \cdot \nabla_{r} F=J(F, F)+\overline{\mathbf{T}} F
$$

where $F(\mathbf{r}, \mathbf{v}, t)$ is the single-particle distribution function for the gas particles, $J(F, F)$ is the nonlinear Boltzmann collision operator, and $\mathbf{T}$ denotes the collision operator that describes the collisions of the gas particles with the macroscopic object placed in the gas. For the case of interest here we consider the object to be a sphere of radius $R$ that is rotating about the $\hat{\mathbf{z}}$ axis with angular velocity $\omega(t)=\omega(t) \hat{\mathbf{z}}$, that can vary with time. We will assume also that $\overline{\mathbf{T}}$ is a linear combination of a specular collision operator and a diffusive collision operator of the form

$$
\overline{\mathbf{T}}=\overline{\mathbf{T}}_{\alpha}=(1-\alpha) \overline{\mathbf{T}}_{\mathrm{sp}}+\alpha \overline{\mathbf{T}}_{\mathrm{di}}
$$

where $\alpha$ is the accommodation coefficient. The operator $\overline{\mathbf{T}}_{\mathrm{sp}}$ has a form identical to that given in Paper $I$, and is unaffected by the rotation of the sphere, while $\overline{\mathbf{T}}_{\mathrm{di}}$ is identical to the form given in I only when considered in a coordinate frame that is attached to the rotating sphere.

As in the previous papers it is convenient to rewrite the extended 
Boltzmann equation in a coordinate frame attached to the sphere. In this frame the gas particles appear to be rotating about the $z$ axis with angular velocity $-\omega(t) \hat{\mathbf{z}}$, and the coordinate transformation to the body fixed system introduces fictitous forces that act on the particles to maintain this rotation. The transformation from the laboratory system to the body fixed system is defined by the relation that ${ }^{(12)}$

$$
\mathbf{v}_{\text {lab }}=\mathbf{v}_{\text {body }}+\omega(t) \times \mathbf{r}
$$

where $\mathbf{v}_{\text {lab }}$ and $\mathbf{v}_{\text {body }}$ are the velocity of a particle in the inertial laboratory frame and in the body fixed frame, respectively. Now we suppose, in addition, that the system is close to a state of total equilibrium in the laboratory frame and that $\omega$ is small, so that we may linearize all quantities in $\omega$. Thus, in the body frame we suppose that $F(\mathbf{r}, \mathbf{v}, \mathbf{t})$ has the form

$$
F(\mathbf{r}, \mathbf{v}, t)=n \phi_{0}(\mathbf{r}, \mathbf{v}, \omega)[W(\mathbf{r})+\Psi(\mathbf{r}, \mathbf{v}, t)]
$$

where $n$ is the number density, $W(\mathbf{r})$ is a function that vanishes for $r<R$ and is unity otherwise, and $\phi_{0}(\mathbf{r}, \mathbf{v}, \boldsymbol{\omega})$ is the Maxwell Boltzmann velocity distribution function in the lab frame, which in the body frame has the form

$$
\begin{aligned}
\phi_{0}(\mathbf{r}, \mathbf{v}, \omega) & =\left(\frac{\beta m}{2 \pi}\right)^{3 / 2} \exp \left\{-\frac{\beta m}{2}[\mathbf{v}+\omega(t) \times \mathbf{r}]^{2}\right\} \\
& \approx \phi_{0}(v)\{1-\beta m \mathbf{v} \cdot[\omega(t) \times \mathbf{r}]\}
\end{aligned}
$$

with $\phi_{0}(v)=(\beta m / 2 \pi)^{3 / 2} \exp -(\beta m / 2) v^{2}$, and in the second line of Eq. (2.5), we have linearized the distribution function in $\omega .{ }^{4}$ If we transform Eq. (2.1) to the body fixed system, and use Eq. (2.4) and (2.5) for the distribution function in this system, we obtain the linearized Boltzmann equation

$$
\frac{\partial \Psi}{\partial t}(\mathbf{r}, \mathbf{v}, t)+\mathbf{v} \cdot \nabla_{r} \Psi(\mathbf{r}, \mathbf{v}, t)-L \Psi-\overline{\mathbf{T}}^{\prime} \Psi=-\mathbf{T}^{\prime} \beta m[\mathbf{v} \cdot(\omega \times \mathbf{r})]
$$

where $\overline{\mathbf{T}}^{\prime}=\left(n \phi_{0}\right)^{-1} \overline{\mathbf{T}}\left(n \phi_{0}\right)$, and $\mathbf{T}^{\prime}=\left(n \phi_{0}\right)^{-1} \mathbf{T}\left(n \phi_{0}\right)$. As in Paper I, we ignore the distinction between $\overline{\mathbf{T}}$ and $\overline{\mathbf{T}}^{\prime}$ and between $\mathbf{T}$ and $\mathbf{T}^{\prime}$ and thus drop the prime notation. To do this we must assume that the sphere and the gas are maintained at the same temperature. The operator $L$ in Eq. (2.6) is the linearized collision operator defined in Paper I. Finally, as we are going to be interested in the quasi-steady-state behavior of the fluid in the vicinity of the sphere and will eventually calculate the torque on the sphere, we take

\footnotetext{
${ }^{4}$ Strictly speaking this linearization is only valid for distances $r$ from the origin such that $(\omega r)^{2} \ll(m \beta)^{-1}$. This restriction will not be a serious constraint for the results to be derived here.
} 
the Laplace transform of Eq. (2.6), and neglect the initial condition term, which describes initial transient effects. This leads to the equation

$$
(\epsilon+\mathbf{v} \cdot \nabla-L-\overline{\mathbf{T}}) \hat{\Psi}(\mathbf{r}, \mathbf{v}, \boldsymbol{\epsilon})=-\mathbf{T} \beta m \mathbf{v} \cdot[\boldsymbol{\omega}(\epsilon) \times \mathbf{r}]
$$

where $\hat{\Psi}(\epsilon)$ is the Laplace transform of $\Psi(t)$, and $\omega(\epsilon)$ is the transform of $\omega(t)$.

As was explained in I and II, it is convenient to separate $\hat{\Psi}$, with the aid of a projection operator, into a hydrodynamical part and a nonhydrodynamical part. The latter is confined to a kinetic boundary layer with an extension of a few mean paths around the surface of the sphere. The following equation can be obtained for the hydrodynamic part of the distribution function:

$$
(\epsilon+\mathbf{v} \cdot \nabla-L-P \tilde{\overline{\mathbf{T}}}) P \hat{\Psi}(\mathbf{r}, \mathbf{v}, \epsilon)=-P \tilde{\mathbf{T}} \beta m \mathbf{v} \cdot[\omega(\epsilon) \times \mathbf{r}]
$$

The $\tilde{\overline{\mathbf{T}}}$ and $\tilde{\mathbf{T}}$ are "renormalized collision operators," which take into account the effects of the nonhydrodynamic distribution function inside the kinetic boundary layer. Their definitions were given in I and II.

The initial source $-P \tilde{\mathbf{T}} \beta m \mathbf{v} \cdot[\omega(\epsilon) \times \mathbf{r}]$ is of the general functional form

$$
v_{\phi} f\left(v_{r}, v_{\theta}, v_{\phi}^{2}, r, \theta\right)
$$

with $v_{\phi}=\mathbf{v} \cdot \hat{\boldsymbol{\phi}} ; \quad v_{\theta}=\mathbf{v} \cdot \hat{\boldsymbol{\theta}} ; \quad \hat{\boldsymbol{\phi}}=(\hat{\mathbf{r}} \times \hat{\mathbf{z}}) / \sin \theta ; \hat{\boldsymbol{\theta}}=-\hat{\mathbf{r}} \times(\hat{\mathbf{r}} \times \hat{\mathbf{z}}) / \sin \theta$ and $\cos \theta=\hat{\mathbf{r}} \cdot \hat{\mathbf{z}}$. This is so because of the symmetry properties of $\tilde{\mathbf{T}}$ and the fact that $\mathbf{v} \cdot[\omega(\epsilon) \times \mathbf{r}]$ is of this functional form. Since the same symmetry properties hold for the operator $(\epsilon+\mathrm{v} \cdot \nabla-L-P(\tilde{\overline{\mathbf{T}}} P)$ occurring on the left-hand side of Eq. (2.8), it follows that the solution $P \hat{\Psi}$ has to be of the same general functional form, as was discussed in I.

There exist only two hydrodynamic distribution functions of the functional form given above, one defined outside and one defined inside the sphere. They are of the general form

$$
\begin{aligned}
\hat{\Phi}_{i}(\mathbf{r}, \mathbf{v}, \epsilon)= & \frac{\delta n_{i}}{n}(\mathbf{r}, \epsilon)+\beta m \mathbf{v} \cdot \mathbf{u}_{i}(\mathbf{r}, \epsilon)+\left(\frac{1}{2} \beta m v^{2}-\frac{3}{2}\right) \frac{\delta T_{i}}{T}(\mathbf{r}, \epsilon) \\
& +A\left(v^{2}\right) \mathbf{v} \cdot \frac{\nabla T_{i}}{T}(\mathbf{r}, \epsilon)+B\left(v^{2}\right)\left(\mathbf{v} \mathbf{v}-\frac{1}{3} v^{2} \mathbf{l}\right): \nabla \mathbf{u}_{i}(\mathbf{r}, \epsilon)+\cdots
\end{aligned}
$$

with the fluid fields $\mathbf{u}_{i}, n_{i}$, and $T_{i}$ given by the solutions of the Laplace transformed linear hydrodynamic equations with a momentum source term 
proportional to $(\hat{\mathbf{z}} \times \hat{\mathbf{r}}) \delta(r-R)$. They are

$$
\begin{aligned}
\hat{\Phi}_{1}: \mathbf{u}_{1} & =u_{0}(\hat{\mathbf{z}} \times \hat{\mathbf{r}}) \theta(r-R) \frac{R^{2}}{r^{2}} e^{(R-r) / \rho} \frac{1+r / \rho}{1+R / \rho} \\
\frac{\delta n_{1}}{n} & =\frac{\delta T_{1}}{T}=0 \\
\hat{\Phi}_{2}: \mathbf{u}_{2} & =u_{0}(\hat{\mathbf{z}} \times \hat{\mathbf{r}}) \theta(R-r) \frac{R^{2}}{r^{2}} e^{(r-R) / \rho} \frac{(1-r / \rho)-(1+r / \rho) e^{-2 r / \rho}}{(1-R / \rho)-(1+R / \rho) e^{-2 R / \rho}} \\
\frac{\delta n_{2}}{n} & =\frac{\delta T_{2}}{T}=0
\end{aligned}
$$

Here $u_{0}$ is an arbitrary constant and $\rho=(\sqrt{\nu / \epsilon})^{1 / 2}$, with $\nu$ the kinematic viscosity; $\nu=\eta / m n$. The function $\theta(x)$ is the unit step function which equals unity for $x \geqslant 1$ and is zero for $x<0$. The solution $P \hat{\Psi}$, of $(2.8)$ must be of the general form $P \hat{\Psi}=\lambda_{1} \hat{\Phi}_{1}+\lambda_{2} \hat{\Phi}_{2}$, since this is the most general hydrodynamic distribution function of the form (2.9).

In order to compute the torque on the sphere, we first follow the method of I.5 and I.6 to obtain $P \hat{\Psi}$ to leading order in the Knudsen number. $P \hat{\Psi}$ may be considered to be generated by a source at the boundary of the sphere. This may be made explicit by a rearrangement of (2.8) as

$$
P \hat{\Psi}=[P(\epsilon+\mathbf{v} \cdot \nabla-L) P]^{-1} S
$$

with

$$
S=P \tilde{\overline{\mathbf{T}}} P \hat{\Psi}-P \tilde{\mathbf{T}} \beta m\{\mathbf{v} \cdot[\boldsymbol{\omega}(\epsilon) \times \mathbf{r}]\}
$$

The inverse operator in (2.12) is defined within the space of hydrodynamic distribution functions. Since $P \hat{\Psi}$ in our case is a linear combination of just two independent distribution functions, the source $S$ can be decomposed likewise as a linear combination of just two independent sources, which, as discussed in $I$, are of the general form $(\mathbf{v} \cdot \hat{\boldsymbol{r}}) \delta(r-R)\left\{\xi_{1} \hat{\Phi}_{1}-\xi_{2} \hat{\Phi}_{2}\right\}$, where $\xi_{1}, \xi_{2}$ are some constants.

As in $I$ the decomposition of the sources is facilitated by passing to a different basis $\hat{\Psi}_{1}, \hat{\Psi}_{2}$ for the hydrodynamic distribution functions, defined as

with

$$
\begin{aligned}
& \hat{\Psi}_{1}=\hat{\Phi}_{1} \\
& \hat{\Psi}_{2}=\hat{\Phi}_{2}+C \hat{\Phi}_{1}
\end{aligned}
$$

$C=\frac{-(1+R / \rho)\left\{-3+3 R / \rho-(R / \rho)^{2}+\left[3+3 R / \rho+(R / \rho)^{2}\right] e^{-2 R / \rho}\right\}}{\left[3+3 R / \rho+(R / \rho)^{2}\right]\left[1-R / \rho-(1+R / \rho) e^{-2 R / \rho}\right]}$ 
The function $\hat{\Psi}_{2}$ has been constructed such that, to zeroth and first order in $(l / R)$, the radial components of the currents of particles, momentum and energy following from $\hat{\Psi}_{2}$ are continuous at the surface of the sphere. It follows directly from (2.10) and (2.11) that the radial currents of mass, energy, and momentum in the $\hat{\boldsymbol{r}}$ and $\hat{\boldsymbol{\theta}}$ directions all vanish for $\Psi_{1}$ and $\Psi_{2}$. Hence, the only nontrivial condition satisfied by $\hat{\Psi}_{2}$ is

$$
\left\langle m(\mathbf{v} \cdot \hat{\mathbf{r}})(\mathbf{v} \cdot \hat{\phi})\left(\hat{\Psi}_{2}^{\mathrm{ext}}-\hat{\Psi}_{2}^{\mathrm{int}}\right)\right\rangle=0
$$

Here $\hat{\Psi}_{2}^{\text {ext }}$ and $\hat{\Psi}_{2}^{\text {int }}$ are the limits of $\hat{\Psi}_{2}$ as the surface of the sphere is approached from the outside and the inside, respectively, and the brackets denote an average weighted with the equilibrium distribution function.

The distribution function $P \hat{\Psi}$ is then of the general form $P \hat{\Psi}$ $=\lambda_{1} \hat{\Psi}_{1}+\lambda_{2} \hat{\Psi}_{2}$ and will be represented as a two-component vector $\left(\lambda_{1}\right)$. The basic sources $S_{1}$ and $S_{2}$ can now be put in the form $(\mathbf{v} \cdot \hat{\mathbf{r}}) \delta(r-R)\left[\mu_{1} \hat{\Psi}_{1}+\right.$ $\left.\mu_{2}\left(\hat{\Psi}^{\mathrm{ext}}-\hat{\Psi}_{2}^{\mathrm{int}}\right)\right]$ and they are chosen such that

$$
\begin{gathered}
\left\langle m(\mathbf{v} \cdot \hat{\boldsymbol{\phi}}) S_{1}\right\rangle=\delta(r-R) \sin \theta \\
{[P(\epsilon+\mathbf{v} \cdot \nabla-L) P]^{-1} S_{1}=\hat{\Psi}_{1} B_{11}} \\
S_{2}=(\mathbf{v} \cdot \hat{\mathbf{r}}) \delta(r-R)\left\{\hat{\Psi}_{2}^{\mathrm{ext}}-\hat{\Psi}_{2}^{\mathrm{int}}\right\}
\end{gathered}
$$

Equation (2.18) states that $S_{2}$ is the exact source of $\hat{\Psi}_{2}$, Eq. $(2.17 \mathrm{~b})$ states that $S_{1}$ generates exclusively the hydrodynamic distribution function $\hat{\Psi}_{1}$, with a strength $B_{11}$, and $(2.17 \mathrm{a})$ determines the value of this constant. Multiplying $(2.17 \mathrm{~b})$ by $m(\mathrm{v} \cdot \hat{\phi}) P(\epsilon+\mathrm{v} \cdot \nabla-L) P$, inserting the result from (2.14a), (2.10), and (2.11a) for $\hat{\Psi}_{1}$ and performing the average implied in (2.17a), one obtains

$$
B_{11}=\frac{R(1+R / \rho)}{u_{0} \eta\left[3+3 R / \rho+(R / \rho)^{2}\right]} .
$$

Putting $B_{22}=1$ and $B_{12}=B_{21}=0$, one may interpret $B_{i j}$ as a $2 \times 2$ matrix, such that $B_{i j}$ describes the amount of $\hat{\Psi}_{i}$ generated by $S_{j}$.

To evaluate the source $S$, as given by (2.13), it is necessary to know the action of the operator $P \tilde{\overline{\mathrm{T}}} P$ on a distribution function of the form $\lambda_{1} \hat{\Psi}_{1}+$ $\lambda_{2} \hat{\Psi}_{2}$. Since $P \tilde{\overline{\mathbf{T}}} P \hat{\Psi}$ is a hydrodynamic source of the general form $\mu_{1} S_{1}+$ $\mu_{2} S_{2}$ one may represent the action of this operator on the $\hat{\Psi}_{i}$ by a $2 \times 2$ matrix A, such that $A_{i j}$ is the amount of $S_{i}$ generated by $P \overline{\mathbf{T}} P$ acting on $\hat{\Psi}_{j}$. From (2.16), (2.17a), and (2.18) it follows that the matrix elements $A_{i j}$ can be determined from the equation

$$
A_{1 j} \sin \theta \delta(r-R)=\left\langle m(\mathbf{v} \cdot \hat{\boldsymbol{\phi}}) P \tilde{\overline{\mathbf{T}}} P \hat{\Psi}_{j}\right\rangle
$$

By the arguments of $I$, Appendix C, the elements $A_{i j}$ are found to be of the 
form

$$
A_{11}=-\gamma u_{0}, \quad A_{12}=-C \gamma u_{0}
$$

to leading order in $l / R$, with $\gamma$ some undertermined constant. ${ }^{5}$ The matrix elements $A_{2 j}$ cannot be determined, but it will turn out that those are not really needed.

What is finally needed for a solution of (2.12) is a determination of the initial source $S^{0}=-P \tilde{\mathbf{T}} \beta m\{\mathbf{v} \cdot[\omega(\epsilon) \times \mathbf{r}]\}$. As in $I$ it is necessary to split up $S^{0}$ according to

$$
S^{0}=-P \tilde{\overline{\mathbf{T}}} \beta m\{\mathbf{v} \cdot[\boldsymbol{\omega}(\boldsymbol{\epsilon}) \times \mathbf{r}]\}+(\mathbf{v} \cdot \hat{\mathbf{r}}) \delta(r-R)\{\mathbf{v} \cdot[\boldsymbol{\omega}(\boldsymbol{\epsilon}) \times \mathbf{r}]\}
$$

The reason for this separation is that the action of $P \tilde{\overline{\mathbf{T}}}$ can be expressed in terms of the unknown constant $\gamma$ appearing in (2.21). Neither $\tilde{\overline{\mathbf{T}}}$ nor $\tilde{\mathbf{T}}$ is known completely, but their difference is, as is made explicit in (2.22). One obtains the component of $S^{0}$ along $S_{1}$, like the matrix elements $A_{i j}$, by multiplying $S^{0}$ by $m(\mathbf{v} \cdot \hat{\boldsymbol{\phi}})$ and averaging over the equilibrium distribution. As a result $S^{0}$ can be written as

$$
S^{0}=\gamma \omega R S_{1}+\mu_{2} S_{2}
$$

to leading order in the Knudsen number, with $\mu_{2}$ some unknown constant.

One can now rewrite (2.12) as a matrix equation

$$
(1-\mathrm{BA}) P \hat{\Psi}=\mathrm{BS}^{0}
$$

where the components of $S^{0}$ along $S_{1}$ and $S_{2}$ are combined into a two-dimensional vector, denoted likewise as $S^{0}$. Equation (2.24) still contains a number of undetermined elements, namely, the components $A_{i j}$ of the matrix A and the component of $S^{0}$ along $S_{2}$. This indeterminancy is removed by imposing the physical condition that $P \hat{\Psi}$ has a zero component along $\hat{\Psi}_{2}$ since the actual distribution function has to vanish inside the sphere. If we represent $P \hat{\Psi}$ as $P \hat{\Psi}=\left(\begin{array}{c}\lambda_{1} \\ 0\end{array}\right)$ and $S_{0}=\left(\begin{array}{c}\omega \omega R \\ \mu_{2}\end{array}\right)$ the equations for $\lambda_{1}$ resulting from (2.24) are

$$
\begin{aligned}
\left(1-B_{11} A_{11}\right) \lambda_{1} & =B_{11} \gamma \omega R \\
A_{21} \lambda_{1} & =\mu_{2}
\end{aligned}
$$

With (2.21) and (2.19), Eq. (2.25a) is solved as

$$
\lambda_{\mathrm{I}}=\frac{\gamma \omega R^{2}(1+R / \rho)}{u_{0}\left\{\eta\left[3+3 R / \rho+(R / \rho)^{2}\right]+\gamma R(1+R / \rho)\right\}}
$$

${ }^{5}$ To derive Eq. (2.21) we used the fact that the computation of $\tilde{\bar{T}} \Psi$ requires the limit of $\Psi$ as the sphere is approached from point $r$, with $r>R$. 
whereas (2.25b) merely produces a relation between the two unknown constants $A_{21}$ and $\mu_{2}$, which has to be satisfied on physical grounds.

The value of the unknown constant $\gamma$ can be calculated explicitly in the case that $\overline{\mathbf{T}}=\overline{\mathbf{T}}_{\alpha}=(1-\alpha) \overline{\mathbf{T}}_{\mathrm{sp}}+\alpha \overline{\mathbf{T}}_{\mathrm{di}}$, with $\alpha$ on the order $l / R$. In that case $^{6}$ (see I.6.15)

$$
\gamma=\alpha n\left(\frac{m}{2 \pi \beta}\right)^{1 / 2}
$$

Substitution into (2.26) yields

$$
\lambda_{1}=\frac{R \alpha\left(R / l_{p}\right)(1+R / \rho)}{u_{0}\left[3+3 R / \rho+(R / \rho)^{2}+\alpha\left(R / l_{p}\right)(1+R / \rho)\right]} \omega(\epsilon)
$$

where we introduced $l_{v}=(2 \pi \beta m)^{1 / 2} v$. The source $S$ of $P \hat{\Psi}$ is then obtained as

$$
S=\mathrm{B}^{-1} P \hat{\Psi}=\frac{\alpha\left(R / l_{\nu}\right)\left[3+3 R / \rho+(R / \rho)^{2}\right]}{3+3 R / \rho+(R / \rho)^{2}+\alpha\left(R / l_{\nu}\right)(1+R / \rho)} \eta \omega(\epsilon) S_{1}
$$

and the torque exerted on the sphere is found to be

$$
\begin{aligned}
\mathbf{M} & =-S \hat{\mathbf{z}} \int_{0}^{\pi} d \theta 2 \pi R^{3} \sin ^{3} \theta \\
& =-\frac{(8 / 3) \pi R^{3} \alpha\left(R / l_{\nu}\right)\left(3+3 R / \rho+(R / \rho)^{2}\right) \eta}{\left[3+3 R / \rho+(R / \rho)^{2}+\alpha\left(R / l_{\nu}\right)(1+R / \rho)\right]} \omega(\epsilon)
\end{aligned}
$$

In the limit $\alpha \rightarrow 0$ the rotation of the sphere has no influence on the fluid. This is in agreement with the fact that specular collisions transfer no tangential momentum to the sphere, and as a result a rotating sphere experiences no friction. On the other hand, when $\alpha$ becomes large compared to $l / R$, Eqs. (2.19), (2.26), and (2.30) reduce to leading order in $l / R$ to the hydrodynamic results for stick-boundary conditions, as obtained by Landau and Lifshitz. ${ }^{(4,8)}$ For $\alpha$ of $O(l / R)$, Eq. (2.30) agrees with the results given by Hynes et al. ${ }^{(10)}$ and by Lebenhaft and Kapral. ${ }^{(11)}$

Instead of using the method of I.5 and I.6 one may as well use the method of I.7. There the continuum limit was taken and explicit calculations were made for both the external and internal distributions resulting from sources containing either a $\delta$ function or the derivative of a $\delta$ function at the surface of the sphere. If we apply this method to the present case, then there is a source of momentum in the $\hat{\phi}$ direction of $\delta$-function type

\footnotetext{
${ }^{6}$ If $\alpha$ is of order $(l / R)$, then $\tilde{\overline{\mathbf{T}}}$ is identical to $\overline{\mathrm{T}}$ up to corrections of order $(l / R)^{2}$.
} 
denoted by $S_{1}^{c}$. In addition a momentum source $S_{2}^{c}$ of the form $S_{2}^{c}=\nabla$. $(\hat{\mathbf{r}} \hat{\dot{\phi}}+\hat{\phi} \hat{\mathbf{r}}) \sin \theta \delta(r-R)$ occurs. As in $I .7$ the superscript $c$ denotes the continuum limit. Either source will generate a certain amount of $\hat{\Psi}_{2}$ in addition to $\hat{\Psi}_{1}$, in this formalism. This can be summarized in the form of a $(2 \times 2)$ matrix $\mathrm{B}^{c}$, such that $B_{i j}^{c}$ is the amount of $\hat{\Psi}_{i}$, generated by $S_{j}^{c}$. The explicit form of this matrix is found by the methods of $I .7$ as

$$
\mathrm{B}^{c}=\left[\begin{array}{cc}
\frac{1}{2 u_{0} \eta} C_{1}(1-C) & 0 \\
\frac{1}{2 u_{0} \eta} C_{1} & \frac{1}{2 u_{0} \eta} C_{2}
\end{array}\right)
$$

where $\eta$ is the coefficient of shear viscosity, and $C_{1}$ and $C_{2}$ are given by

$$
\begin{aligned}
C_{1} & =\left(\rho^{2} / R\right)\left[1-\rho / R+e^{-2 R / \rho}(1+\rho / R)\right](1+R / \rho) \\
C_{2} & =-(\rho / R)\left(1+3 \rho / R+3 \rho^{2} / R^{2}\right)\left[(1-R / \rho)-(1+R / \rho) e^{-2 R / \rho}\right] \\
& =2 /(1-C)
\end{aligned}
$$

and with $C$ given by (2.15). Similarly the action of $\tilde{\overline{\mathbf{T}}}_{\alpha}$ on $\hat{\Psi}_{1}$ and $\hat{\Psi}_{2}$ can be summarized again in the form of a $(2 \times 2)$ matrix $\mathrm{A}^{c}$, such that $A_{i j}^{c}$ is the amount of $S_{i}^{c}$, generated by $\tilde{\overline{\mathbf{T}}}_{\alpha}$ acting on $\hat{\Psi}_{j}$. An explicit calculation similar to (I.7), yields

$$
\mathrm{A}^{c}=\left(\begin{array}{cc}
-\xi n u_{0}\left(\frac{m}{2 \pi \beta}\right)^{1 / 2} & -C \xi n u_{0}\left(\frac{m}{2 \pi \beta}\right)^{1 / 2} \\
-\eta u_{0} & -C \eta u_{0}
\end{array}\right)
$$

with $C$ given by (2.15) and $\xi$ is an unknown positive constant. The initial source is given by (2.22), but now its components along both $S_{1}^{c}$ and $S_{2}^{c}$ have to be found explicitly. The first term on the right-hand side of (2.22) gives a contribution similar to that of $P \tilde{\overline{\mathbf{T}}} \hat{\Psi}_{1}$. Its explicit form, in the usual vector notation reads

$$
\omega R\left(\begin{array}{c}
\xi n\left(\frac{m}{2 \pi \beta}\right)^{1 / 2} \\
\eta
\end{array}\right)
$$

The contribution of the second term on the right-hand side of (2.17) can be calculated likewise by taking the inner product with $\left.\mid \Psi^{u}\right)$, defined in (I.4.11) The result, in vector notation reads $-\omega R\left(\begin{array}{l}0 \\ \eta\end{array}\right)$. Hence $S^{0}$ is given to leading order in $l / R$ as

$$
S^{0, c}=\omega R\left(\begin{array}{c}
\frac{\xi \eta}{l_{v}} \\
0
\end{array}\right)
$$


The resulting complete source follows again from the equivalent of Eq. (I.7.21),

$$
S^{c}=\left(I-\mathrm{A}^{c} \mathrm{~B}^{c}\right)^{-1} S^{0, c}
$$

The distribution function generated by the rotation of the sphere is obtained as

$$
\hat{\Psi}^{c}=\mathbf{B}^{c} S^{c}
$$

After some algebra, one finds the explicit results

$$
\left.\begin{array}{c}
S_{c}=-\frac{R \eta \xi}{l_{\nu}} \omega(\epsilon)\left[\begin{array}{c}
\frac{3+3 R / \rho+(R / \rho)^{2}}{3+3 R / \rho+(R / \rho)^{2}+\left(\alpha R / l_{\nu}\right)(1+R / \rho)} \\
-\frac{R(1+R / \rho)}{\left(\alpha R / l_{\nu}\right)(1+R / \rho)+3+3 R / \rho+(R / \rho)^{2}}
\end{array}\right] \\
\hat{\Psi}^{c}=\frac{R^{2} \xi}{u_{0} l_{\nu}} \omega(\epsilon)\left[\frac{(1+R / \rho)}{\left(\alpha R / l_{\nu}\right)(1+R / \rho)+3+3 R / \rho+(R / \rho)^{2}}\right. \\
0
\end{array}\right]
$$

Here the result (2.28) for $\hat{\Psi}$ is recovered. Furthermore an amount of $S_{2}^{c}$ is generated, which compensates for the amount of $\hat{\Psi}_{2}$, generated by $S_{1}^{c}$, in contrast to $S_{1}$. However, the amounts of $\hat{\Psi}_{1}$ generated by $S_{1}$ and $S_{1}^{c}$ are equal, which explains the equality of their coefficients in (2.29) and (2.37). The torque exerted on the sphere is not influenced by the occurrence of $S_{2}^{c}$, since this source does not contribute to the torque.

\section{THE ROTATING CYLINDER}

The case of an infinitely long cylinder rotating about its axis, or equivalently the case of a disk rotating about its center in a twodimensional gas, can be treated by the same methods as the rotating sphere. Unlike the case of a cylinder performing translational motion, ${ }^{(2)}$ no divergences arise from the linearization of the distribution function about the Maxwellian in the rest frame of the cylinder. Thus we do not have to use the trick of linearizing the distribution function about its value in the rest frame of the fluid (see II). ${ }^{7}$ For simplicity we restrict ourselves to the method of (I.5 and I.6) as discussed in Section 2 for the sphere. All calculations are completely analogous to those given for the sphere. The

\footnotetext{
${ }^{7}$ The reason is that in the rotating-cylinder case the velocity field decays by one power of $1 / r$ faster for large $r$ than in the case of translational motion.
} 
hydrodynamic distribution functions that are rotationally symmetric about the $z$ axis are of the form (2.5) with the fluid fields for the outside solution defined as ${ }^{8}$

$$
\begin{aligned}
\hat{\Psi}_{1}: \mathbf{u}_{1} & =u_{0}(\hat{\mathbf{z}} \times \hat{\mathbf{r}}) \frac{K_{1}(r / \rho)}{K_{1}(R / \rho)} \theta(r-R) \\
\frac{\delta n_{1}}{n} & =\frac{\delta T_{1}}{T}=0
\end{aligned}
$$

Here $K_{1}$ is a Bessel function of the second kind. ${ }^{(13)}$ In addition there is a distribution $\hat{\Psi}_{2}$, defined such that through order $l / R$, the radial currents of particles, momentum, and energy vanish for $\hat{\Psi}_{2}$. The precise form of this function is not needed here. For the hydrodynamic sources, we choose again a source $S_{1}$, satisfying the analog of $(2.17 \mathrm{a}, \mathrm{b})$, and a source $S_{2}$, satisfying the analog of (2.18). Next we define matrices $B$ and $A$ again, such that $B_{i j}$ is the amount of $\hat{\Psi}_{i}$ generated by $S_{j}$, and $A_{i j}$ is the amount of $S_{i}$ generated by $P \tilde{\overline{\mathrm{T}}} P$ acting upon $\hat{\Psi}_{j}$. As was seen from (2.19), the only important elements of these matrices are $A_{11}$ and $B_{11}$. These are readily calculated as

$$
\begin{aligned}
& A_{11}=\frac{\gamma \eta}{l_{v}} \\
& B_{11}=\frac{R}{\eta u_{0}} \frac{K_{1}(R / \rho)}{(R / \rho) K_{2}(R / \rho)}
\end{aligned}
$$

To leading order in the Knudsen number the unknown constant $\gamma$ is the same as for the sphere, since, on the length scale of the mean free path both a sphere and a cylinder appear as a flat plate to lowest approximation. The initial source is given by (2.22) again and, as there, the second term on the right-hand side does not contribute to $S_{1}$ and the whole initial source can be represented by the analog of (2.23),

$$
S^{0}=\gamma \omega R S_{1}+\mu_{2} S_{2}
$$

If we require again that the distribution function vanishes inside the cylinder, we may obtain the distribution function and its source from (2.25a) and (2.29) as

$$
\begin{gathered}
\hat{\Psi}=\frac{\alpha R^{2}}{u_{0} l_{v}} \omega(\epsilon) \frac{K_{1}(R / \rho)}{(R / \rho) K_{2}(R / \rho)+\left(\alpha R / l_{v}\right) K_{1}(R / \rho)} \hat{\Psi}_{1} \\
S=\frac{\alpha R \eta}{l_{v}} \omega(\epsilon) \frac{(R / \rho) K_{2}(R / \rho)}{(R / \rho) K_{2}(R / \rho)+\left(\alpha R / l_{\nu}\right) K_{1}(R / \rho)} S_{1}
\end{gathered}
$$

${ }^{8}$ We take the cylinder axis to be in the $z$ direction. 
The torque per unit length exerted on the cylinder is then obtained as

$$
\mathbf{M}=-2 \pi R^{2} S \hat{\mathbf{z}}
$$

We want to make a few remarks concerning these results. First of all in the limit $\rho \rightarrow \infty$ (or $\epsilon \rightarrow 0)$ the Bessel functions can be expanded ${ }^{(13)}$ as

$$
\begin{aligned}
& K_{1}(x)=\frac{1}{x}+\frac{1}{2} x\left(\ln \frac{x}{2}+\mathbf{c}-\frac{1}{2}\right)+O\left(x^{3}\right) \\
& K_{2}(x)=\frac{2}{x^{2}}-\frac{1}{2}+O\left(x^{2}\right)
\end{aligned}
$$

where $\mathbf{c}$ is Euler's constant.

If we substitute this into the result for $\mathbf{M}$ and examine the limiting case $\alpha \gg l_{v} / R$, we find

$$
\mathbf{M}=-4 \pi \eta R^{2}\left[1-\frac{R^{2}}{2 \rho^{2}}\left(\ln \frac{R}{2 \rho}+\mathbf{c}\right)+O \frac{R}{\rho}^{4}\right] \omega(\epsilon) \hat{\mathbf{z}}
$$

For the stationary case this is in agreement with the existing literature. ${ }^{(14)}$ For the nonsteady case (3.9) can be transformed back into time language, yielding an expression of the form

$$
\mathbf{M}(t)=-\int_{0}^{t} d \tau \zeta(\tau) \omega(t-\tau) \hat{\mathbf{z}}
$$

where $\zeta(t)$ is the time-dependent rotational friction kernel. From (3.9) it follows that $\zeta(t)$ has a long time tail proportional to $t^{-2}$, while the translational friction kernel decays as $t^{-1}$ for long times. ${ }^{(2)}$ This is in agreement with the observation that rotational friction kernels decay by one power $1 / t$ faster than the corresponding translational friction kernel. ${ }^{(4,6,8)}$ The same holds for the diffusion kernel ${ }^{9}$ of a particle performing translational or rotational Brownian motions. ${ }^{(9)}$ This follows immediately from the fact that the diffusion kernels are connected to the friction kernels by Stokes-Einstein relations of the type $\mathrm{e}^{(9,10,15)}$

$$
\hat{C}(\epsilon)=\beta^{-1}[\epsilon \mu+\zeta(\epsilon)]^{-1}
$$

Here $\hat{C}(\epsilon)$ is the Laplace transform of either the velocity autocorrelation function or the angular momentum autocorrelation function of the Brownian particle. Then $\mu$ is either the mass or the moment of inertia of the Brownian particle and $\hat{\zeta}$ is the translational or the rotational friction coefficient, respectively.

\footnotetext{
${ }^{9}$ The diffusion kernel is identical to the velocity autocorrelation function in the case of translational diffusion and to the angular momentum autocorrelation function in the case of rotational diffusion.
} 


\section{CONCLUSION}

We have shown that the methods developed in Papers I and II can also be used to treat the rotation of a sphere or cylinder in a dilute gas. In the case of small Knudsen numbers all the results we obtained were in agreement with hydrodynamics. The method we followed in this paper to solve the extended Boltzmann equation was the generalized normal solution method discussed in I. In II, however, we showed that this method is completely equivalent to an iterative solution method. Hence the latter could have been used just as well.

As mentioned in the Introduction, the work described here is closely related to the work of other authors on rotational Brownian motion. ${ }^{(9-11)}$ It is, therefore, of considerable interest to provide a kinetic-theory treatment of Brownian motion in order to describe both translational and rotational diffusion of the Brownian particle in terms of microscopic collision dynamics. Such a kinetic theory description of Brownian motion involves calculations closely related to the iterated solution of the extended Boltzmann equation presented in Paper II, where the molecule-object collisions are taken into account explicitly. Progress in this direction has been made recently by a number of authors who have discussed the translational diffusion of a Brownian particle. In particular, Cukier et al. ${ }^{(16)}$ and Keyes and Mercer ${ }^{(17)}$ have shown that the dynamical events considered in II, the ring and repeated ring events involving the Brownian particle and the molecules of the surrounding gas, lead to an expression for the translational diffusion kernel given by Eq. (3.11) with the proper hydrodynamic friction coefficient. More recently, Mercer ${ }^{(18)}$ has considered the translational Brownian motion of a heavy Brownian particle moving in a low-density gas, and has shown how the Fokker-Planck equation can be derived from the repeated ring kinetic equation for the Brownian particle. However, a complete kinetic theory for Brownian motion of a particle in a moderately dense gas is likely to be considerably more complicated, since dynamical processes not included in the extended Boltzmann will have to be taken into account. We intend to address some of these points in Paper IV of this series.

\section{REFERENCES}

1. H. van Beijeren and J. R. Dorfman, J. Stat. Phys. 23:335 (1980).

2. H. van Beijeren and J. R. Dorfman, J. Stat. Phys. 23:443 (1980).

3. J. R. Dorfman and H. van Beijeren, in Statistical Physics, Part B, B. Berne (ed.), Plenum Press, New York (1978); see also J. H. Ferzigen and H. G. Kaper, Mathematical Theory of Transport in Gases, North-Holland, Amsterdam (1972).

4. L. Landau and E. M. Lifshitz, Fluid Mechanics, Pergamon Press, New York (1954).

5. H. Lamb, Hydrodynamics, Dover, New York (1945). 
6. Y. Pomeau and P. Resibois, Phys. Rep. 19C:64 (1975).

7. J. T. Hynes, Ann. Rev. Phys. Chem. 28:301 (1977).

8. S. Yip, Ann. Rev. Phys. Chem. 30:547 (1979).

9. E. Hauge and A. Martin-Löf, J. Stat. Phys. 7:259 (1973).

10. J. T. Hynes, R. Kapral, and M. Weinberg, J. Chem. Phys. 67:3256 (1977).

11. J. R. Lebenhaft and R. Kapral, J. Chem. Phys. 74:6888 (1981).

12. Cf, L. Landau and E. M. Lifshitz, Mechanics, 3rd ed., Pergamon Press, New York (1975), pp. 126-129.

13. Cf. I. S. Gradshteyn and I. M. Ryzhik, Tables of Integrals, Series and Products, Academic Press, New York (1965).

14. Cf. H. Lamb, Hydrodynamics, Dover, New York (1945), p. 588. The torque on a cylinder rotating in a rarefied gas has been computed by A. Gervois and Y. Pomeau, Phys. Fluids 17:2292 (1974).

15. J. T. Hynes and J. M. Deutch, in Physical Chemistry: An Advance Treatise, Vol. IIB, H. Eyring, D. Henderson, and W. Jost (eds.), McGraw-Hill, New York (1975); see also N. Corngold, Phys. Rev. A 6:1570 (1972).

16. R. I. Cukier, R. Kapral, J. R. Lebenhaft, and J. R. Mehaffey, J. Chem. Phys. 73:5244 (1980).

17. T. Keyes and J. M. Mercer (private communication).

18. J. M. Mercer, The kinetic theory of Brownian motion, Ph.D. dissertation, Yale University (1981). 